\title{
Management of Severe Bilateral Ptosis in a Patient With Midbrain Infarction: A Case Report
}

\author{
Soo Yeon Kim, MD, Hye Kyung Park, MD, Dae Heon Song, MD, \\ Myung Eun Chung, MD, Young Moon Kim, MD, Jae Hyun Woo, MD
}

Department of Rehabilitation Medicine, St. Paul's Hospital, The Catholic University of Korea College of Medicine, Seoul, Korea

Ptosis could be caused by oculomotor nerve palsy in the midbrain infarction. Bilateral ptosis has been reported in several reports, which focused on clinical characteristics of midbrain infarction. Little research attention has been paid to the treatment of patients with bilateral ptosis in midbrain infarction. We experienced a case of severe bilateral ptosis occurring after midbrain infarction. The patient could not open her eyes, perform basic activities or achieve effective rehabilitation. Neurogenic ptosis can improved after the underlying cause is treated. However, in this case, bilateral ptosis was not improved after conservative care for 6 months and the patient remained limited in activities of daily living and mobility. Surgical correction of bilateral ptosis was done by the resection of both Muller's muscles. After surgical correction, the bilateral ptosis was much improved and the effect persisted for at least 6 months.

Keywords Blepharoptosis, Cerebral infarction, Oculomotor nerve diseases

\section{INTRODUCTION}

The midbrain is supplied by the posterior cerebral artery (PCA), basilar artery, superior cerebellar artery, and anterior choroidal artery. The midbrain is often affected in patients with embolic stroke occurring in the posterior circulation, usually with the concomitant involvement of other structures, such as the pons, thalamus, and the cer-

Received November 27, 2012; Accepted May 15, 2013

Corresponding author: Myung Eun Chung

Department of Rehabilitation Medicine, St. Paul's Hospital, The Catholic University of Korea College of Medicine, 180 Wangsan-ro, Dongdaemungu, Seoul 130-709, Korea

Tel: +82-2-958-2307, Fax: +82-2-968-2307, E-mail: coltrane@catholic.ac.kr

(c) This is an open-access article distributed under the terms of the Creative Commons Attribution Non-Commercial License (http://creativecommons. org/licenses/by-nc/3.0) which permits unrestricted noncommercial use, distribution, and reproduction in any medium, provided the original work is properly cited.

Copyright ( 2013 by Korean Academy of Rehabilitation Medicine ebellum. Infarction limited to the midbrain is rare. The reported prevalence of pure midbrain infarction varies from $0.7 \%$ to $2.3 \%[1,2]$.

Ptosis could be caused by oculomotor nerve palsy in the patients with midbrain infarction. In several cases, bilateral ptosis showed the clinical characteristics of midbrain infarction $[1,3,4]$. However, these reports focused on the correlation between clinical and radiological findings. Little attention has been paid to the treatment of patients with bilateral ptosis in midbrain infarction.

We experienced a case of severe bilateral ptosis that occurred after midbrain infarction in which the patient could not open her eyes and was limited in basic activities and mobility. Almost 6 months after the onset of bilateral ptosis, symptoms had not improved at all. Surgical correction of bilateral ptosis and management had considerable benefit. We herein report the case with a review of relevant literature. 


\section{CASE REPORT}

A 73-year-old woman with a previous history of hypertension and myocardial infarction was admitted to the neurosurgery department via the emergency room because she could not open her eyes and had altered consciousness. She was diagnosed as acute infarction on the bilateral paramedian midbrain including bilateral cerebral peduncle by magnetic resonance imaging (Fig. 1).

After conservative management, her conscious level improved but she was still unable to open her eyes. Examination revealed pronounced bilateral ptosis, with left and right pupil diameter of $6 \mathrm{~mm}$ and $3 \mathrm{~mm}$, respectively. The left pupil was not reactive to light. There was marked limitation of adduction, moderate limitation of depression and elevation in the movement of both eyeballs. Abduction was normal. There were no other findings of central nervous system disease that could cause ptosis from brain imaging, and no evidence of other orbital disease causing ptosis in an ophthalmologic examination. Muscle strength rated using the Medical Research Council Scale was grade $3 / 5$ in both upper limbs and $4 / 5$ in both lower limbs. She was transferred to the department of rehabilitation medicine for intensive rehabilitation management. Participation in the rehabilitation programs was difficult due to continuing inability to open her eyes.

We tried taping her lids with Micropore tape and used lubricants to prevent exposure keratopathy. However, this method was less efficient due to blinking. Eye-putti eyelash glue-mediated fixation of the upper eyelid to the supraorbital structures was ineffective. The patient became frustrated and depressed, and refused to partici- pate in the rehabilitation programs.

After about 6 months from the stroke attack, bilateral ptosis was not improved at all. Consultations were held with the department of plastic surgery with the aim of considering surgical treatment, with the thinking that it would be hard to anticipate spontaneous recovery. Plastic surgeons planned the surgical correction of bilateral ptosis and the Muller's muscle procedure was done, considering the benefits and losses.

After surgical correction, the upper lid height was improved from 7.0 to $4.0 \mathrm{~mm}$ on the right side and from 7.0 to $4.5 \mathrm{~mm}$ on the left side. The palpebral fissure height was $3.5 \mathrm{~mm}$ on the right side and $3.0 \mathrm{~mm}$ on the left side (Fig. 2). The patient was quite contented with the effect of the operation and actively participated in the rehabilitation programs. She was able to perform activities of daily living and mobility with minimal assistance. The effect of surgical correction has persisted for at least 6 months.

\section{DISCUSSION}

Paramedian midbrain infarction results from occlusion of the branches (the medial mesencephalic branch $[\mathrm{MMB}])$ that diverge from the proximal portion of the PCA before the branching of the posterior communicating artery [3]. The MMB is divided into the superior MMB, which supplies the rostral part of the midbrain, and the inferior MMB, which supplies the caudal part of the midbrain. Bilateral midbrain infarction usually produces alterations in the level of consciousness, cognitive abnormalities, behavioral abnormalities and eye movement disorders [4]. Eye movement disorders include ocu-
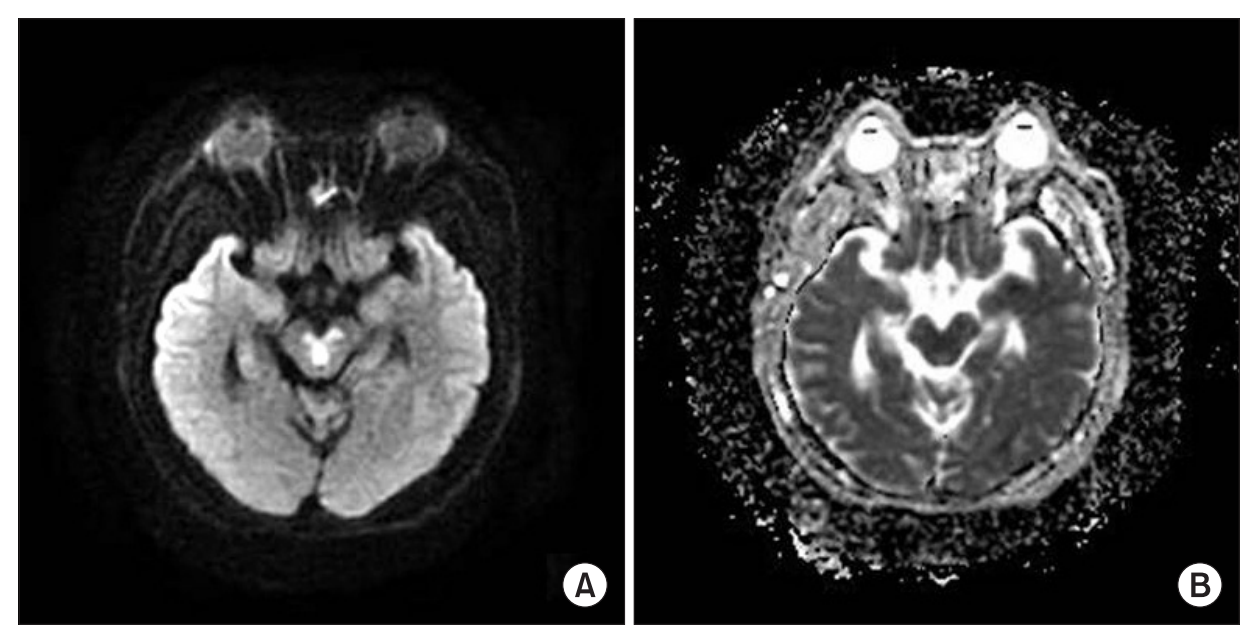

Fig. 1. Brain diffusion-weighted imaging and apparent diffusion coefficient map at clinical onset. (A) Axial diffusion-weighted imaging demonstrates increased signal in the bilateral paramedian midbrain. (B) Apparent diffusion coefficient map demonstrates hypointense signal in corresponding areas, consistent with restricted diffusion. 


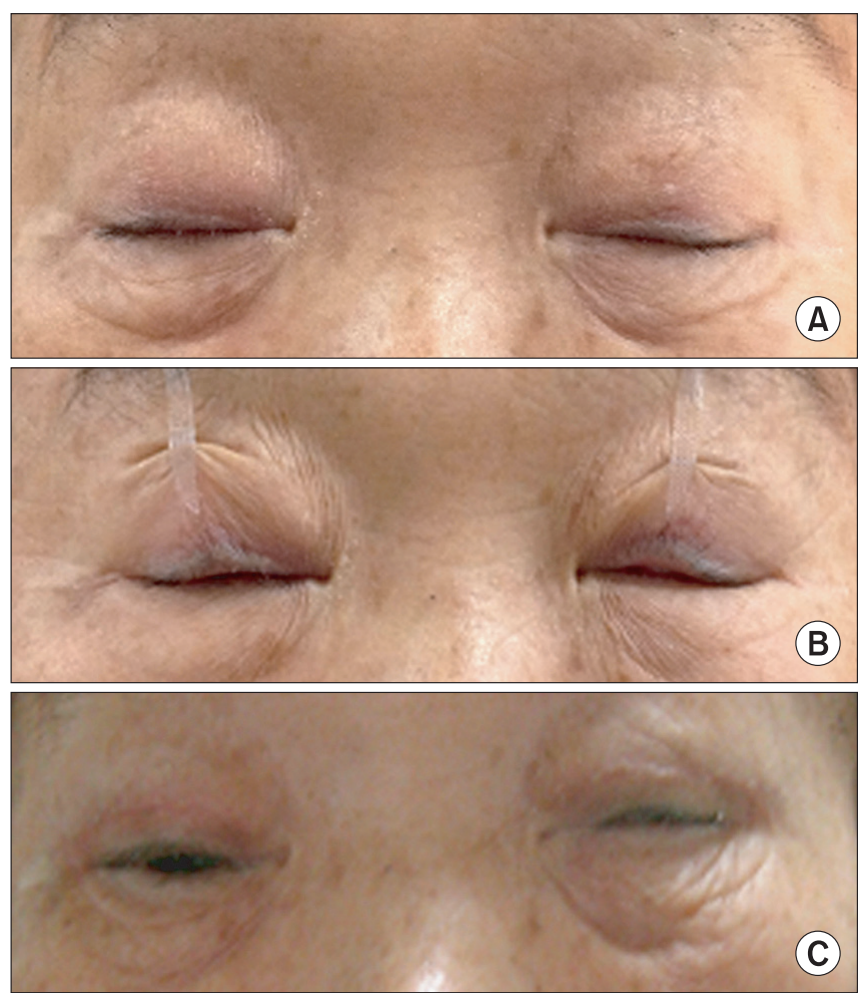

Fig. 2. Follow-up photographs of the patient's eyelids. (A) Before surgery, the patient had complete bilateral ptosis. Ptosis was $7 \mathrm{~mm}$ and the levator function was $0 \mathrm{~mm}$ on both eyes. The palpebral fissure height was uncheckable. (B) We tried to tape the eyelids using Micropore tape. However, this method was less efficient due to the blinking mechanism. (C) After surgery, the ptosis was improved by $4 \mathrm{~mm}$ on the right side and $4.5 \mathrm{~mm}$ on the left side. The palpebral fissure height was $3.5 \mathrm{~mm}$ on the right side and $3.0 \mathrm{~mm}$ on the left side.

lomotor nerve palsy, vertical gaze palsy, and internuclear ophthalmoplegia.

The nucleus of the oculomotor is located at the ventromedial part of the central gray in the rostral midbrain and consists of the lateral somatic cell column, caudal central nucleus, and medial cell column. The Edinger-Westphal nucleus is called the accessory oculomotor nerve nucleus and is located at the dorsomedial region of the oculomotor nerve nucleus [5].

In our case, ocular symptoms were manifested by bilateral oculomotor nerve palsies. Abnormal movement of extraocular muscles implies involving the lateral and medial cell column, which regulates extraocular muscles including the inferior rectus, inferior oblique, medial rectus and superior rectus muscles. The abnormality of the left papillary function implies involvement of the left Edinger-Westphal complex, which leads the parasympathetic nerve fibers to the sphincter pupillae muscles and ciliary muscles over the ciliary ganglion. Bilateral ptosis is the hallmark of involvement of the central caudal nucleus, which regulates the levator palpebrae superioris muscle on both sides.

Bilateral ptosis was reported in a few reports that presented the clinical characteristics of midbrain infarction. In 2005, Kim and Kim [1] reported the clinical findings of 40 patients with pure midbrain infarction. Ocular motor manifestations were seen in 21 (53\%) patients. Among the 40 patients, 12 had ptosis, which was unilateral in 10 patients and bilateral in two patients. In 2012, Ogawa et al. [3] reported a clinical study of 21 patients with pure midbrain infarction. Ptosis was present in four patients. Ptosis was unilateral in three patients and bilateral in only one patient. Most patients with bilateral ptosis as a manifestation of midbrain infarction had poor prognosis because the affected midbrain lesions were multiple and broad. In 2008, Thurtell and Halmagyi [4] reported three cases of complete ophthalmoplegia, the combination of bilateral ptosis with loss of all extraocular movements as a manifestation of bilateral paramedian midbrainthalamic infarction. Among these patients, two had died. In a previous study conducted by Ogawa et al. [3], the patient presenting the bilateral ptosis had bilateral multiple (rostral and caudal) midbrain infarction and a drowsy level of consciousness. There has been little attention paid to the management of the patients with bilateral neurogenic ptosis in midbrain infarction.

Ptosis may be classified according to various criteria, such as etiology, severity, and levator function. Based on etiology, ptosis has myogenic, neurogenic, aponeurotic, mechanical or traumatic causes [6]. Neurogenic ptosis is due to the malfunction or damage of the oculomotor or sympathetic nerve, or central nervous system abnormalities. Other conditions that frequently affect the oculomotor nerve are diabetes, tumors, aneurysms, multiple sclerosis, intoxication with heavy metals and trauma. Usually, neurogenic ptosis can be corrected and may spontaneously resolve if the underlying cause is treated. Therefore, time should be allowed for any possible recovery of function before surgical intervention. But, in a stroke, such as our case, it is not easy to correct the cause of ptosis and the degree of recovery will vary depending 
on the severity of initial brain damage and recovery. In previously reported cases of ptosis with infarction, the prognosis for recovery of ptosis was poor $[4,5]$. According to severity, the degree of ptosis can be classified as mild (1-2 mm), moderate (3-4 mm), or severe (>4 mm). The amount of ptosis is measured as the distance between the upper lid height and the upper limbus and lid margin. According to the levator function, the degree ptosis can be classified as poor $(0-4 \mathrm{~mm})$, moderate $(5-10 \mathrm{~mm})$, or good (>10 mm). The levator function expressed as the distance between the excursion of the upper lid margin from full downward gaze to full upward gaze.

In our case, the upper lid height was $7 \mathrm{~mm}$ and the levator function was $0 \mathrm{~mm}$ on both eyes before surgery. The severity of ptosis was severe with poor levator function. Palpebral fissure height could not be checked because the patient could not open her eyes at all. Although the patient showed slight improvement in ocular adduction, bilateral ptosis was not improved after 6 months from stroke attack.

Nonsurgical conservative treatment of ptosis included eye-putti eyelash glue or glasses made with a crutch attachment to hold up the lid [7]. We tried compensatory methods that could hold up the lid using tape and glue, but these methods could lead to a corneal injury because the patient kept the tape on all day and were less efficient due to the inevitable blinking that occurred. For these reasons, surgical correction was considered for the patient's quality of life.

The type of surgical procedure is determined according to the severity of ptosis and levator function. Severe ptosis with a poor levator function usually requires some type of brow/frontalis suspension. However, in this case, there was no Bell's phenomenon, which is an important protective reflex wherein the globe is turned upwards and slightly outwards during the eye closure. The serious complication of exposure keratitis can be developed due to poor Bell's phenomenon postoperatively [7]. van Sorge et al. [8] showed the risk and incidence of exposure keratopathy following silicone frontalis suspension in adult ptosis with poor Bell's phenomenon. According to these authors, overall exposure keratopathy occurred in $26 \%$. Exposure keratopathy including punctate epithelial erosion and corneal ulceration is also associated with excessive corneal exposure in cases with overcorrected ptosis. Therefore, in this case, although the effect of surgical cor- rection would be weak, Muller's muscle procedure, which was usually recommended for mild ptosis was done. This method is performed by resecting the conjunctiva and Muller's muscle via the posterior approach and it is a frequently applied procedure because of its ease, precision and predictability. Complications that may occur after surgery include under-, over-correction, infection, bleeding, and reduced vision.

In our case, upper lid height was improved by $4.0 \mathrm{~mm}$ on the right side and $4.5 \mathrm{~mm}$ on the left side after surgery. The palpebral fissure height was $3.5 \mathrm{~mm}$ on the right side and $3.0 \mathrm{~mm}$ on the left side. There was no complication after surgery. The aim of the surgery was not aesthetic improvement but to help the patient perform the basic activities independently by opening the eyes. After surgery, the patient became active in a rehabilitation program. Improvement in the visual acuity and field of vision also resulted in improvements in mobility and balance. Although bilateral ptosis was not completely corrected and the eyelids may not appear perfectly symmetrical, our purpose was achieved.

Surgical correction could be a good treatment option for neurogenic ptosis, when neurologic recovery does not occur spontaneously. However, when selecting the degree or type of surgical procedure, careful consideration of the benefit and loss is needed.

\section{CONFLICT OF INTEREST}

No potential conflict of interest relevant to this article was reported.

\section{REFERENCES}

1. Kim JS, Kim J. Pure midbrain infarction: clinical, radiologic, and pathophysiologic findings. Neurology 2005;64:1227-32.

2. Bogousslavsky J, Maeder P, Regli F, Meuli R. Pure midbrain infarction: clinical syndromes, MRI, and etiologic patterns. Neurology 1994;44:2032-40.

3. Ogawa K, Suzuki Y, Oishi M, Kamei S. Clinical study of twenty-one patients with pure midbrain infarction. Eur Neurol 2012;67:81-9.

4. Thurtell MJ, Halmagyi GM. Complete ophthalmoplegia: an unusual sign of bilateral paramedian midbrain-thalamic infarction. Stroke 2008;39:1355-7. 
5. Donzelli R, Marinkovic S, Brigante L, Nikodijevic I, Maiuri F, de Divitiis O. The oculomotor nuclear complex in humans: microanatomy and clinical significance. Surg Radiol Anat 1998;20:7-12.

6. Finsterer J. Ptosis: causes, presentation, and management. Aesthetic Plast Surg 2003;27:193-204.

7. Betharia SM, Kalra BR. Observations on Bell's phe- nomenon after levator surgery. Indian J Ophthalmol 1985;33:109-11.

8. van Sorge AJ, Devogelaere T, Sotodeh M, Wubbels R, Paridaens D. Exposure keratopathy following silicone frontalis suspension in adult neuro- and myogenic ptosis. Acta Ophthalmol 2012;90:188-92. 